\title{
Raman Lidar Observations: Simultaneous Measurements of Water Vapor, Temperature and Aerosol Vertical Profiles, Part I
}

\author{
Takashi Shibata, Tetsu SaKaI, Masahiko Hayashi, Tetsuro OJo, \\ Soung-An KwON, and Yasunobu IWASAKA
}

\author{
Solar-Terrestrial Environment Laboratory, Nagoya University, Furo-cho, Chikusa-ku, Nagoya 464-01, Japan
}

(Received April 24, 1995; Revised June 25, 1995; Accepted March 7, 1996)

\begin{abstract}
A Raman lidar was developed to observe vertical profiles of water vapor, temperature and aerosols. The lidar system can detect signal from 5 detectors simultaneously. Two vibrational Raman backscattering echoes from $\mathrm{H}_{2} \mathrm{O}$ and $\mathrm{N}_{2}$ (or $\mathrm{O}_{2}$ ) excited by Nd:YAG 3 rd harmonics wavelength $(355 \mathrm{~nm})$ are used to observe the profiles of water vapor mixing ratio and atmospheric temperature (or density). Nd:YAG fundamental and 2nd harmonics wavelengths (1064 and $532 \mathrm{~nm}$ ) are used to observe backscattering coefficient profiles of aerosols and clouds at these wavelengths. The scattering at $532 \mathrm{~nm}$ is observed in parallel and perpendicular components corresponding to the linear polarization plane of the transmitted linearly-polarized laser pulse to observe depolarization ratio profiles. Observed water vapor and temperature distributions coincide with the simultaneously observed radiosonde sounding data excellently. The Raman lidar makes it possible to observe the vertical profiles of water vapor, temperature, aerosols, and clouds simultaneously.
\end{abstract}

\section{Introduction}

Humidity data are fundamental information for studying atmospheric science. There are several methods to measure humidity. The radiosonde sounding is the standard method to observe vertical profiles of humidity in the troposphere. However, the measurements are only accurate to within $10 \%$ at best. With this technique it is difficult to observe short time variations of the vertical profiles.

As a new equipment to observe vertical distribution of water vapor in the troposphere, the Raman lidar was developed by several lidar groups (e.g. Melfi et al., 1969; Vaughan et al., 1988; Eichinger et al., 1994; Jackson and Gasiewski, 1995). In Raman lidar observation, laser light is Raman shifted for special wavenumber in each molecule by vibrational Raman scattering. The recent development of a high energy laser with a shorter wavelength in near ultraviolet region makes it easy to use this technique to observe water vapor. The Raman lidar can observe only the limited species of atmospheric constituents because the scattering coefficient of Raman scattering is orders of magnitude smaller than that of Rayleigh or Mie scattering (e.g. Inaba, 1976). However, it can give us very useful information about atmospheric species because of its high temporal and spatial resolution, and simultaneous multi-component observation capability.

The Mie scattering lidar is very powerful tool to observe aerosol and cloud profiles. In studying tropospheric aerosols by the lidar technique, the existence of water liquid or ice particles makes it difficult to estimate the background amount of aerosol particles. Since water molecules are one of the main constituents of aerosol particles, determining water vapor concentration is important in studying tropospheric aerosol properties. Needless to say, cloud formation is essentially controlled by water vapor concentration. However, in almost all cases, humidity data was not taken simultaneously with Mie lidar observations until now.

A new lidar system was developed to investigate the relation between tropospheric particles and water vapor. In this system both Raman scattering from a few atmospheric constituents and Mie/Rayleigh scattering by aerosol particles and atmospheric molecules are simultaneously measured. The new lidar 
makes it possible to obtain vertical profiles of particles, water vapor and temperature at the same time. Using both of Mie/Rayleigh and Raman scattering lidar should give us much information that would advance aerosol research, as well as cloud physics.

In this paper and an accompanying paper we will describe the performance of the Raman lidar system and data retrieving techniques that we developed. In Part I, the hardware of the lidar system is discussed, along with some observed results, as well as the results of the comparison with radiosonde sounding. In the accompanying paper (Part II) we present our method of analysis and the characteristics of the observed data, and discuss the accuracy of the final results.

\section{Lidar Equipment and Observations}

The Raman lidar transmitter uses three wavelengths from a conventionalNd:YAG laser: fundamental (F) wavelength $1064 \mathrm{~nm}$, second harmonic generation (SHG) $532 \mathrm{~nm}$, and third harmonic generation (THG) $355 \mathrm{~nm}$. The output energy of each wavelength is respectively $150 \mathrm{~mJ} /$ pulse for $\mathrm{F}, 150 \mathrm{~mJ} / \mathrm{pulse}$ for SHG, and $300 \mathrm{~mJ} /$ pulse for THG. The pulse repetition rate of the laser is 10 pulses per second (pps). All the wavelengths are linearly polarized. The laser beam emitted through the lidar transmitter is collimated to about $0.2 \mathrm{mrad}$.

A schematic diagram of the receiving portion of the lidar system is shown in Fig. 1. Light backscattered by the atmosphere with Raman, Rayleigh and Mie scattering processes are focused by a one meter diameter telescope. Since the telescope is pointed upwards, the lidar only observes in a fixed vertical

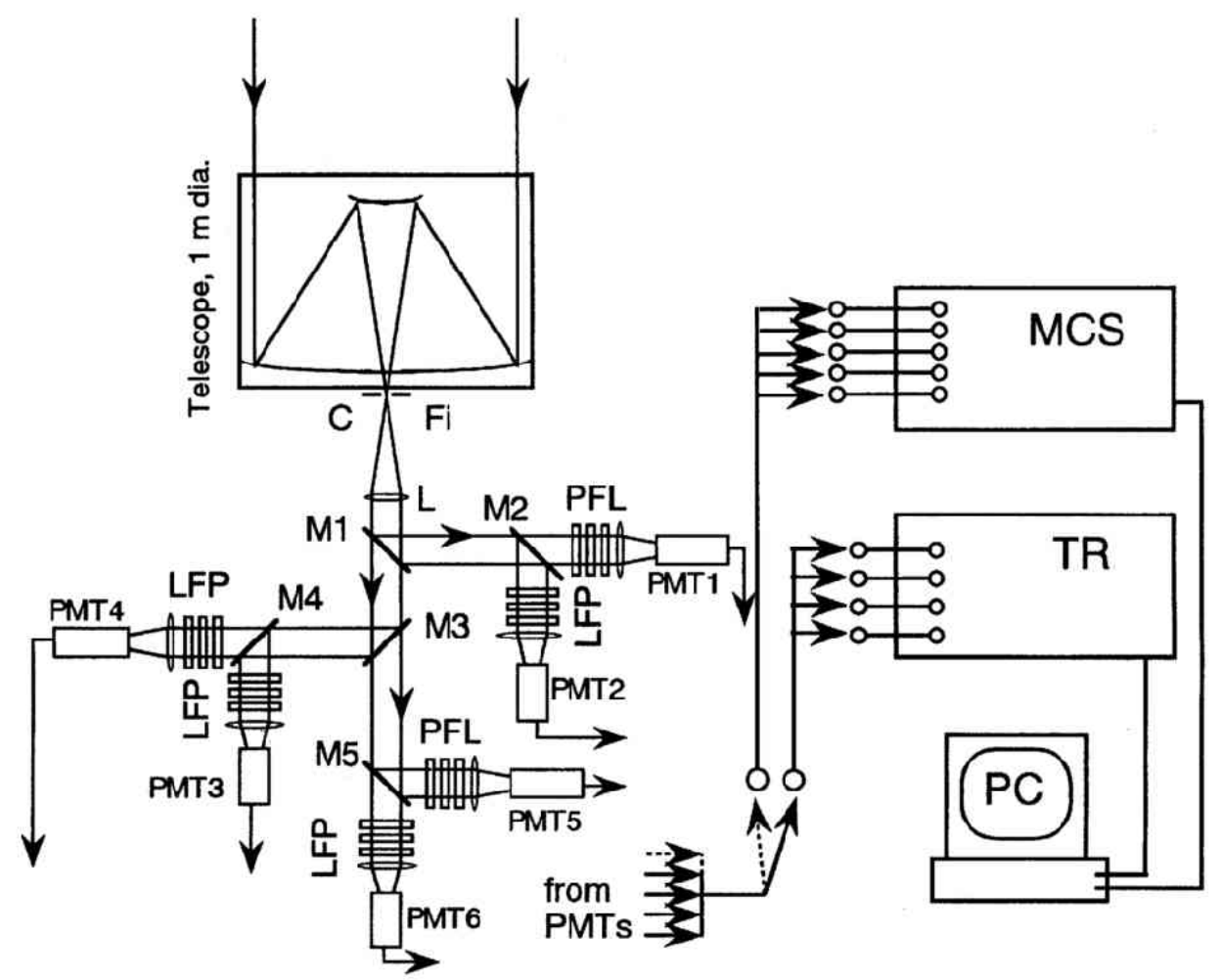

Fig. 1. Schematic diagram of the Raman lidar receiver optical system, $(C=$ Chopper; $F i=$ field stop; $L=$ lens; $M 1-5=$ mirrors; $\mathrm{F}=$ interference filter, Rayleigh cut filter, or neutral density filter; $\mathrm{P}=$ polarizer; $P M T 1-6=$ photomultiplier tubes). The arrows entering the telescope and running to the PMTs indicate the optical path. The arrows extending from the PMTs indicate the electrical lines. 
direction. The signal light is divided into six photomultiplier tubes (PMT) through a chopper, mirrors, interference filters and polarizers. All the PMTs are cooled to $-30^{\circ} \mathrm{C}$ to decrease thermal noise.

The electrical signal from the PMTs is processed by either five multichannel scalers (MCS) in photon counting mode, or four transient recorders (TR) in analog mode. The five or four PMTs are arbitrarily connected with these MCSs or TRs depending on the observation mode. Vertical spatial resolution is 50 $\mathrm{m}$ or $100 \mathrm{~m}$ in photon counting mode, and $1.5 \mathrm{~m}$ or longer in analog mode.

Although the wavelength and polarization detected by each PMT can be selected by changing beam splitting optical components, routine observation is carried out with the following set up: PMT 1 and PMT 2 in Fig. 1 are used for detecting Raman shifted wavelengths excited by the Nd:YAG THG. The wavelength from water vapor is $407.4 \mathrm{~nm}$ (PMT 1) and that from $\mathrm{N}_{2}\left(\mathrm{O}_{2}\right)$ is 386.6 (375.4) nm (PMT 2). The lidar echo from each wavelength is separated by beam splitter mirrors. Rayleigh and Mie scattering contamination by direct THG scattering is eliminated by Rayleigh blocking filters and interference filters. The signal is detected by the PMTs after being transmitted through Rayleigh blocking filters and interference filters. The rejection factor of Rayleigh and Mie scattered THG wavelength is less than $10^{-8}$. The FWHM of IF filter is $1 \mathrm{~nm}$.

PMT 3 and PMT 4 are used for detecting SHG wavelength. PMT 3 is used for detecting parallel polarization component of backscattered signals from transmitted laser polarization, and PMT 4 is used for detecting perpendicular component. These data are used for retrieving depolarization ratio of aerosol particles at $532 \mathrm{~nm}$. Polarization components are selected by the polarizer prism in front of the filters and the PMTs.

PMT 5 is used for detecting F wavelength near infrared region. A low noise photomultiplier tube (HAMAMATSU, R3236; Uchino et al., 1991) is used as PMT 5. The data from the MCSs or TRs are analyzed by personal computer. Characteristics of the Raman lidar are summarized in Table 1.

A radiosonde made by VAISALA was used in the comparative observations with the Raman lidar and the in situ radiosonde. It takes about 1 hour for the sonde to reach the maximum altitude of about 30

Table 1. Characteristics of the Raman lidar system.

\begin{tabular}{cl}
\hline Transmitter & Nd:YAG \\
Laser & $1064,532,355$ \\
Wavelengths (nm) & $300,150,150$ \\
Energy/pulse (mJ) & $10 \mathrm{pps}$ \\
Pulse repetition & $0.2 \mathrm{mrad}$ (after collimator) \\
Laser beam divergence & \\
& 1 m dia. Cassegrain type \\
Receiver & HAMAMATSU R331 (for UV and visible) \\
Telescope & HAMAMATSU R3236 (for IR) \\
PMTs & $355,372,373,375,387,396,407,532,1064$ \\
IF filters & 1 \\
Center wavelengths, & $10^{-6}$ \\
FWHM (nm), & \\
Rejection factor & $<10^{-2}$ \\
Rayleigh/Mie cut filter & \\
Transmission at $355 \mathrm{~nm}$ & 5 \\
MCS & 50 or $100 \mathrm{~m}$ \\
Channel number & $100 \mathrm{MHz}$ \\
Range resolution & \\
Pulse count rate & 4 \\
Transient recorder & $>1.5 \mathrm{~m}$ \\
Channel number & $8 \mathrm{bit}$ \\
Range resolution & \\
Signal resolution & \\
\hline
\end{tabular}


$\mathrm{km}$ from the ground. Lidar observation was performed continuously, including during the flight time of the radiosondes. Lidar data was taken every 5 minutes. Usually, lidar data accumulated for 30 minutes are used to calculate the profiles of water vapor concentration, temperature and aerosols. The data taken in the 30 minutes during the radiosonde flight are used in the comparison.

\section{Results and Discussions}

Routine observation with the Raman lidar began in late 1992. Comparisons with radiosonde soundings were conducted four times in 1994, the results of which are presented in this section. The processing method is described in Part II.

Figure 2 shows the profile of the water vapor mixing ratio observed by Raman lidar on September 10,1994 (solid line) as well as the mixing ratio profile observed simultaneously by radiosonde sounding (broken line). The lidar data are calibrated by least square fitting with radiosonde data between 0.3 and $6 \mathrm{~km}$ in altitude. The two profiles are in good agreement. Figure 2 also shows the mixing ratio observed by lidar that is calculated only from system calibration factors and Raman backscattering coefficient (thin line). As discussed in Part II, there are more than $50 \%$ difference in the cross-sections of Raman backscattering that appear in some papers. This brings the largest uncertainty in the absolute value of the Raman observed water vapor mixing ratio.

The calibration factor is calculated using water vapor profiles observed by radiosonde so that lidar observed data are least square fitted with radiosonde data between 0.3 to $6 \mathrm{~km}$ in altitude. Three other comparative observations show similar agreement based on the same calibration factor. This indicates that

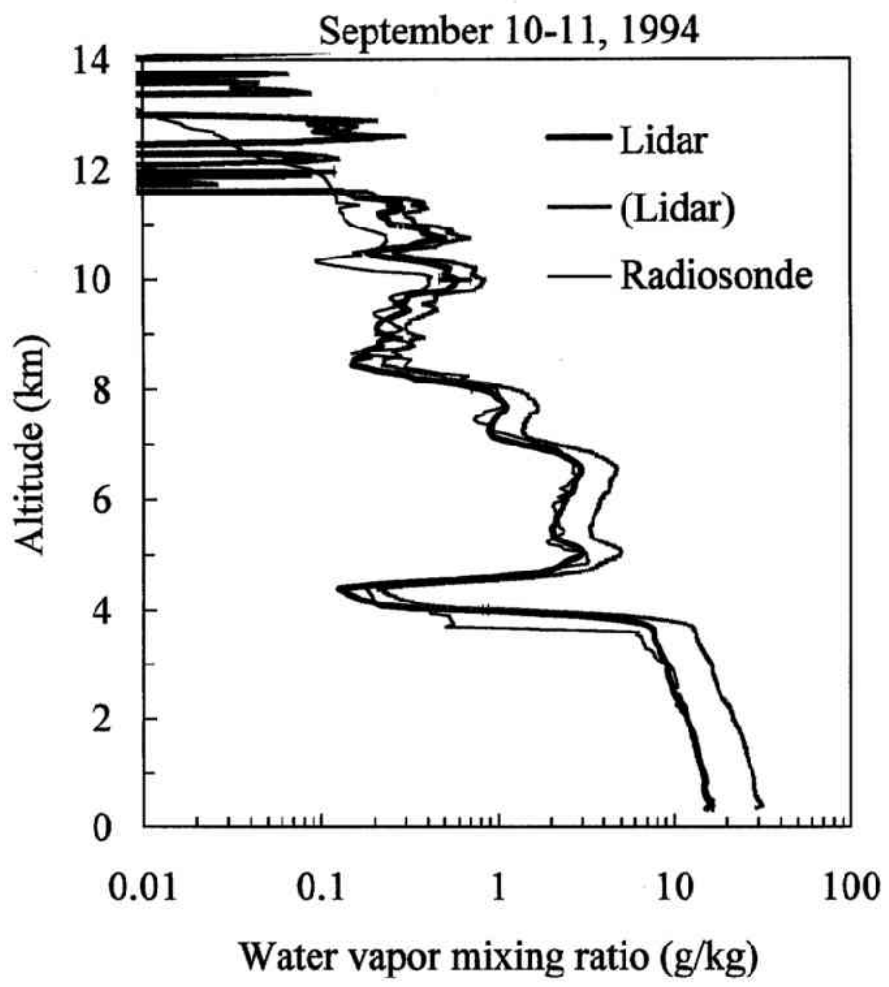

Fig. 2. Profiles of the water vapor mixing ratio on September 10, 1994 observed by Raman lidar (solid lines) and radiosonde (dotted line). The thin line is a lidar observed profile calibrated only with system parameters. 


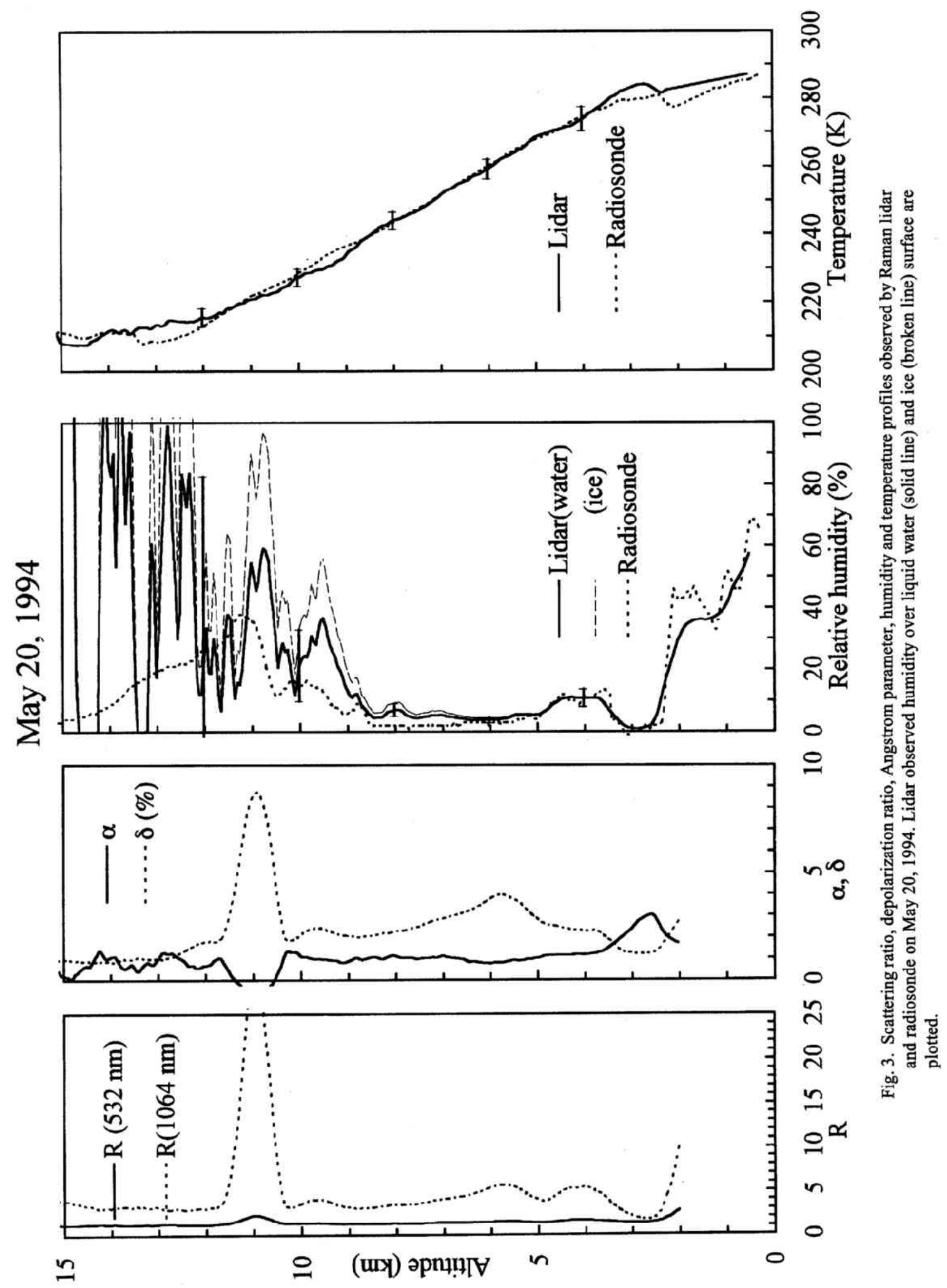


the lidar observations of water vapor are reliable.

Figure 3 shows profiles of the scattering ratio $(R)$ at 532 and $1064 \mathrm{~nm}$, depolarization ratio $(\delta)$ and Angstrom coefficient $(\alpha)$ calculated by backscattering coefficients at F and SHG wavelengths, humidity, and temperature on May 20,1994. The scattering ratio $R$ is defined as

$$
R=\frac{\beta_{\text {Mie }}+\beta_{\text {Rayleigh }}}{\beta_{\text {Rayleigh }}},
$$

where $\beta_{\text {Mie }}$ and $\beta_{\text {Rayleigh }}$ are backscattering coefficients of Mie and Rayleigh scattering. The depolarization ratio $\delta$ is defined as follows;

$$
\delta=\frac{\beta_{S}}{\beta_{P}+\beta_{S}}
$$

where $\beta_{P}$ and $\beta_{S}$ are parallel and perpendicular components of backscattering coefficient. The Angstrom parameter $\alpha$ is the exponent when we assume backscattering coefficient $\beta$ follows the relation

$$
\beta \propto \lambda^{-\alpha},
$$

where $\lambda$ is wavelength. $R-1\left(=\beta_{\text {Mie }} / \beta_{\text {Rayleigh }}\right)$ is roughly proportional to the mass mixing ratio of the particles. $\delta$ is a measure of non-sphericity of particles, and $\alpha$ is a parameter of size distribution. Based on

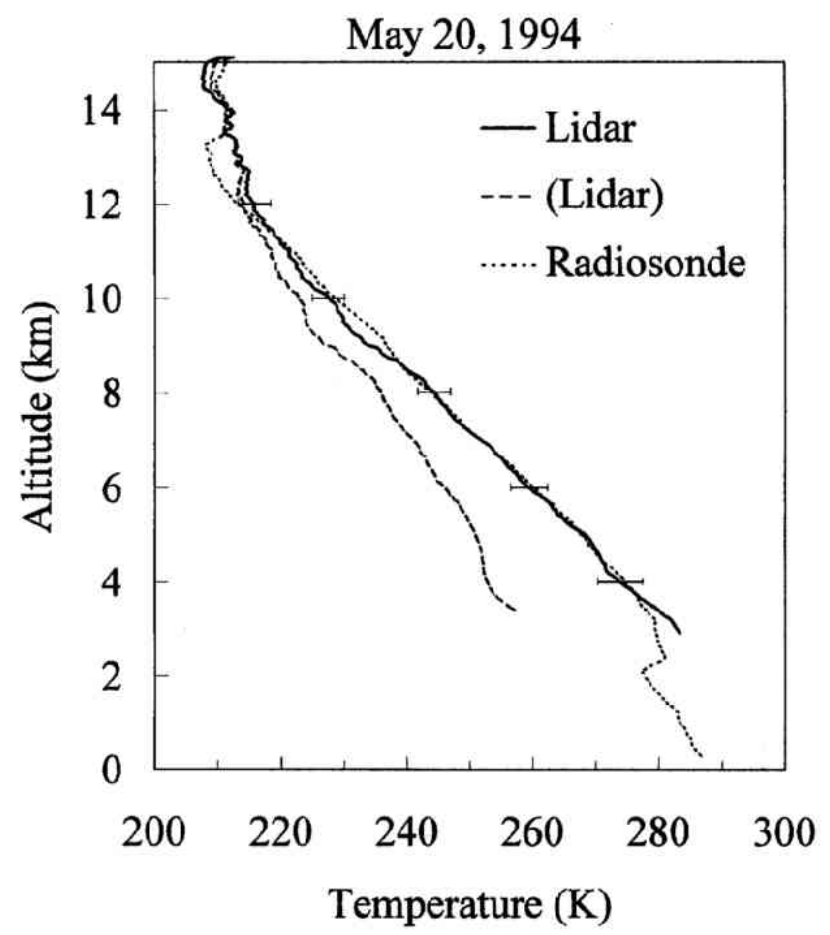

Fig. 4. Temperature profiles on May 20, 1994 observed by Raman lidar (solid lines) and a radiosonde (dotted line). The broken line is a lidar observed profile in which only the extinction by Rayleigh scattering was taken into account. 
these profiles, the relation between temperature, humidity and characteristics of atmospheric particles can be understood from data taken with the Raman lidar.

Clouds exist at altitudes between 10 and $12 \mathrm{~km}$ in the profiles of Fig. 3. Humidity observed by lidar is very high at this altitude range (broken line). Angstrom parameter $\alpha$ is almost 0 at the cloud altitude indicating a larger radius of cloud particles. Depolarization peak indicates the existence of crystalline particles. On the other hand, humidity is very low at the scattering ratio peak at about $6 \mathrm{~km}$ in altitude, but has a maximum at the $R$ peak near $4 \mathrm{~km}$. At $4 \mathrm{~km} \delta$ is smaller than at $6 \mathrm{~km}$.

VAISALA guarantees the accuracy of humidity data at temperature over $-40^{\circ} \mathrm{C}$. The temperature at an altitude higher than about $9 \mathrm{~km}$ is under $-40^{\circ} \mathrm{C}$. This is the altitude at which the discrepancy between the humidity data by the radiosonde and that by the lidar starts to increase. The accuracy of the radiosonde at low temperatures is caused by the humidity sensor's time constant being so long at ambient temperatures lower than $-40^{\circ} \mathrm{C}$ that the radiosonde underestimates observed humidity. Usually, the humidity near clouds is thought to be close to $100 \%$. Although further research is needed to know what makes the discrepancy between the lidar and sonde data, the Raman lidar can give a better estimation of the humidity,

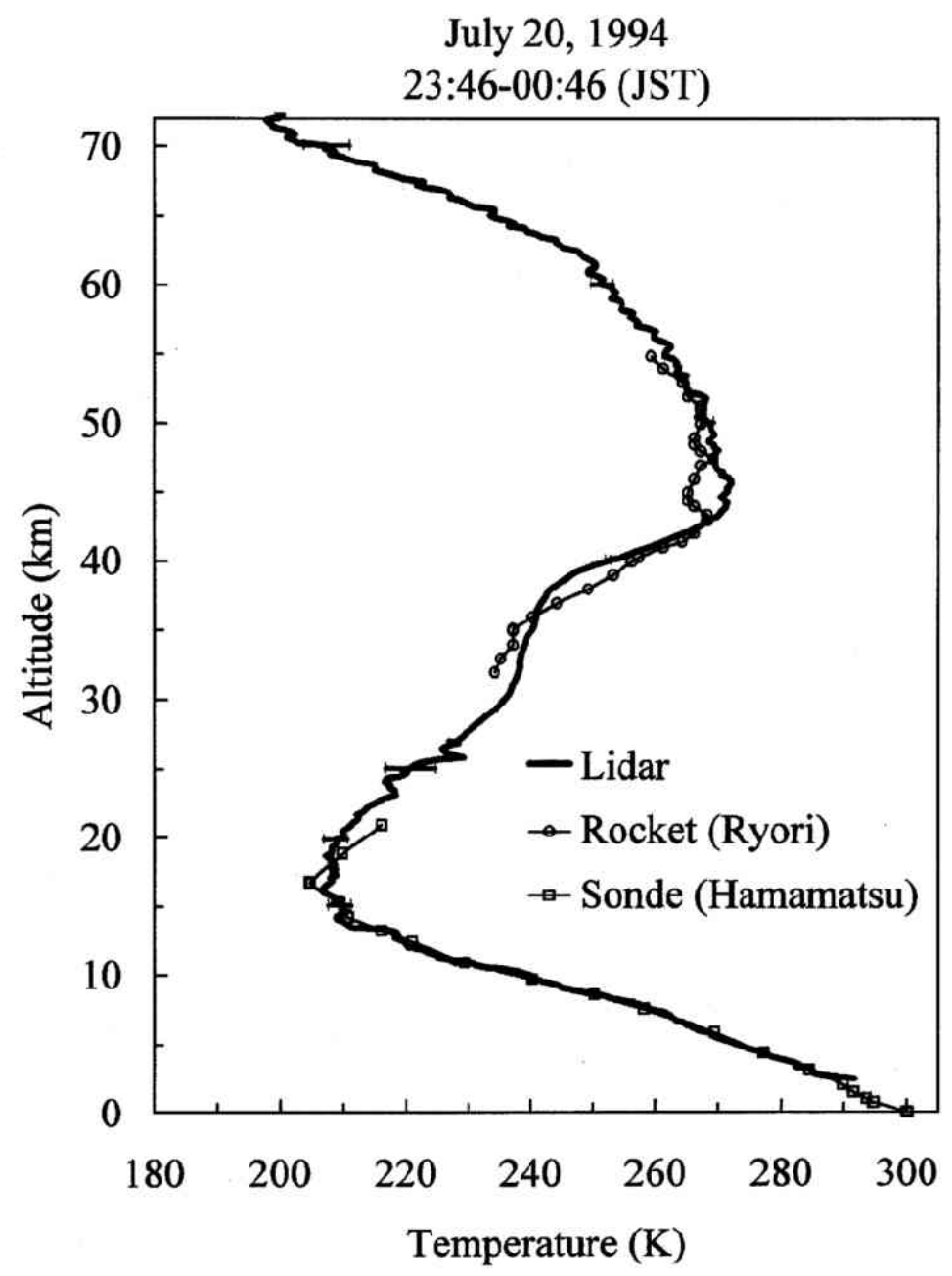

Fig. 5. Temperature profiles on July 20, 1994 observed by Raman lidar, a rocket and a radiosonde. The rocket was launched at Ryori about $500 \mathrm{~km}$ northeast of Nagoya. The radiosonde was launched at Hamamatsu about $50 \mathrm{~km}$ east of Nagoya. 
even if we take into consideration the relative error of the lidar observed humidity being large at the altitude of about $10 \mathrm{~km}$.

Figure 4 shows the temperature profile obtained from the atmospheric density profile by $\mathrm{O}_{2}$ Raman scattering on the night of May 20,1994. The sonde observed profile and the profile in which the extinction effect only by Rayleigh scattering was corrected were also plotted in the figure. The temperature only with the Rayleigh scattering correction was underestimated by about 10 to 20 degrees as compared to the sonde observed temperature. The temperature calculated taking both Rayleigh and Mie extinction into account and the sonde observed temperature are in close agreement. The agreement between the temperature observed by the Raman lidar and by the radio sonde demonstrates the usefulness of vibrational Raman scattering for observing tropospheric temperature profiles if we can simultaneously estimate Mie and Rayleigh extinction effects accurately enough. The method of the calculations and the error of the calculated results are discussed in Part II.

Figure 5 shows the temperature profile for altitudes up to $70 \mathrm{~km}$ observed on July 20,1994 . In lower altitudes from 2 to $10 \mathrm{~km} \mathrm{O}_{2}$ Raman scattering is used, and in the lower stratosphere from 10 to $30 \mathrm{~km} \mathrm{~N}_{2}$ Raman scattering is used. Rayleigh scattering is used for higher altitudes. Observations were made for 30 min. in each altitude range. By connecting Raman scattering and Rayleigh scattering echoes, temperatures from free troposphere to mesopause altitudes can be measured within an error of a few degrees in most of the altitude range under $70 \mathrm{~km}$.

At low altitudes under $10 \mathrm{~km}$ we do not use $\mathrm{N}_{2}$ but instead use $\mathrm{O}_{2}$ Raman scattering for the observations, because, at low altitudes, the $\mathrm{N}_{2}$ Raman echo is so strong that it saturates the signal in our MCS system unless it is attenuated (e.g., by a neutral density filter). The $\mathrm{O}_{2}$ signal is not saturated at this altitude range. Although $\mathrm{N}_{2}$ signal is, of course, also useful, using the $\mathrm{O}_{2}$ signal is the easiest method to observe tropospheric density in our system at the present time. Relative vertical profiles from both $\mathrm{O}_{2}$ and $\mathrm{N}_{2}$ coincide well at altitudes near $10 \mathrm{~km}$.

\section{Concluding Remarks}

The usefulness of vibrational Raman scattering in lidar observation of atmospheric density in the lower atmosphere has been doubted because of the existence of aerosol particles. It is thought to be difficult to correct the extinction by Mie scattering precisely enough (e.g. Nedeljkovic et al., 1993). But, this is not the result of the investigation by experiments with multi-wavelength observations as ours, or even not by the simulation with a detailed optical model. In our observations it was found that this is not the case for many tropospheric conditions.

The Raman lidar system shows excellent performance in observing water vapor in the troposphere, temperature from the troposphere to the mesopause, and aerosols and clouds. The main components of the lidar system are now readily available. However, the simultaneous multi-wavelength detection of Raman and Rayleigh/Mie scattering provides us with new possibilities in studying aerosol and cloud physics, as well as atmospheric dynamics. The simultaneously observed data at the same spatial region will give us new insights into atmospheric science.

\section{REFERENCES}

Eichinger, W. E., D. Cooper, F. L. Archuletta, D. Hof, D. B. Holtkamp, R. R. Karl, Jr., C. R. Quick, and J. Tiee, Development of a scanning, solar-blind, water Raman lidar, Appl. Opt., 18, 3923-3932, 1994.

Inaba, H., Detection of atoms and molecules by Raman scattering and resonance fluorescence, in Laser Monitoring of the Atmosphere, edited by E. D. Hinkley, pp. 153-236, Springer-Verlag, Berlin, 1976.

Jackson, D. M. and A. J. Gasiewski, Millimeter-wave radiometric observations of the troposphere: a comparison of measurements and calculations based on radiosonde and Raman lidar, IEEE TGRS, 33, 3-14, 1995.

Melfi, S. H., J. D. Lawrence, Jr., and M. P. McCormick, Observation of Raman scattering by water vapor in the atmosphere, Appl. Phys. Lett., 15, 295-297, 1969.

Nedeljkovic, D., A. Hauchecorne, and M-L. Chanin, Rotational lidar to measure the atmospheric temperature from the ground to $30 \mathrm{~km}$, IEEE TGRS, 31, 90-101, 1993. 
Uchino, O., H. Takashima, and I. Tabata, Lidar measurements of stratospheric and tropospheric aerosols at $1064 \mathrm{~nm}$ using a new and low-noise photomultiplier, J. Met. Soc. Jpn., 69, 709-714, 1991.

Vaughan, G., D. P. Wareing, L. Tomas, and V. Mitev, Humidity measurements in the free troposphere using Raman backscatter, J. R. Meteorol. Soc., 114, 1471-1484, 1988. 